\title{
What's new for the clinician: excerpts from and summaries of recently published papers
}

SADJ May 2019, Vol. 74 No. 4 p203 - p206

Compiled and edited by $\mathrm{V}$ Yengopal

\section{The Erich arch bar versus embrasure wires for intraoperative intermaxillary fixation in mandibular fractures}

\author{
AS Satpute, SA Mohiuddin, AM Doiphode, et al. Oral Maxillofac Surg. 2018; 22: 419-28.
}

The mandible is the largest and main bone of the lower part of the face. Anatomic parts of the mandible are the symphysis, parasymphysis, body, angle, ramus, coronoid process, condyle, and alveolus. Inherent weak sites of mandible which are liable to fracture include the angle of the mandible (especially when third molar is impacted), the socket of the canine tooth (due to long root of canine, the associated bone amount is reduced), and the condylar neck.

Maxillofacial injuries constitute one of the common and major health problems worldwide. Maxillofacial fractures are often associated with substantial morbidity, deformity, loss of function, and high treatment cost. The important factors to be considered while treating mandibular fractures are reduction, fixation, immobilization, and occlusal stability. The intermaxillary fixation (IMF) provides a stable base, from which facial form and function can be restored. However, in recent practice with the advent of open reduction and internal fixation (ORIF), the use of IMF is now limited intraoperatively.

The arch bar technique has been used since World War I. In spite of its simplicity and reliability, there are numerous problems associated with this technique such as compromised periodontal status, high needle prick injuries, and long treatment time. In 1999, Hollows and Brennan described a simple technique of IMF called "embrasure wire," which they claimed was less time consuming, had low needle prick injuries, and was cost effective. Satpute and colleagues (2018) from India reported on a trial that sought to compare the conventional Erich arch bar with embrasure wire during intraoperative IMF for ORIF in mandibular fractures under general anesthesia.

Veerasamy Yengopal: $B C h D, B S c H o n s, M C h D, P H D$, Community Dentistry Department, School of Oral Health Sciences, University of Witwatersrand, Medical School, no. 7 York Road, Parktown 2193, South Africa. Email address: veerasamy.yengopal@wits.ac.za

\section{ACRONYM}

IMF: Intermaxillary Fixation

ORIF: Open Reduction and Internal Fixation

\section{MATERIALS \& METHODS}

In this prospective, comparative clinical study, 50 dentulous patients with mandibular fractures who required IMF followed by open reduction and internal fixation under general anaesthesia as a part of the treatment plan were selected.

Patients between 18 and 60 years of age with favourable/ unfavourable fractures of the mandible were included in this study. Edentulous patients, patients with primary and mixed dentition, pathologic mandibular fractures, mandibular condylar fracture, maxillary fracture, panfacial fractures, and patients with gross generalized spacing in the dentition were excluded from this study.

Patients were selected by simple randomized sampling and the sample was subsequently divided into group A and group B, each having 25 patients. Group A patients received IMF with Erich arch bars, and group B patients received IMF with embrasure wire.

Patients in Group A received the technique for IMF by using the Erich arch bar. After administration of general anesthesia, a prefabricated arch bar with hooks incorporated on the outer surface of the flat malleable stainless steel metal strip, was cut accurately to the lengths of both upper and lower dental arches.

On the upper jaw, the hooks were arranged in an upward direction and to the lower jaw in a downward direction. The arch bar was adapted to the buccal surface from $1^{\text {st }}$ molar to $1^{\text {st }}$ molar in both arches. It was fixed to each tooth, using prestretched 26-gauge stainless steel wire, which was passed from the mesial surface of the teeth 
to the lingual side and back to the buccal side from the distal surface of the tooth, making sure that one end of the wire passed above the arch bar and the other below it.

After this, both ends of the wire were twisted together in a clockwise manner and the arch bar was thus attached securely and firmly to the necks of each tooth on the buccal surface of the arch.

Open reduction and internal fixation were then carried out Patients in Group B were treated by IMF by using embrasure wires. After administration of general anesthesia, the number and position of the embrasure wires to be inserted was determined according to the type and location of the fracture.

A single prestretched 20 -gauge wire of $15 \mathrm{~cm}$ length was passed through the facial embrasure between the maxillary first molar and first premolar, and then the palatal end of the wire was looped and passed through the opposite lingual embrasure in the mandible. A similar procedure was repeated on the contralateral side to achieve intermaxillary fixation. After securing the occlusion, the ends of the wire were twisted together on the buccal surface of the lower premolar and molar teeth on both sides and intermaxillary fixation was achieved.

For removal, the embrasure wires were untwisted and cut on the facial surface, and pressure was applied on the chin to gently push the mandible away from the maxilla, creating a small gap between mandibular and maxillary dentitions. Then, the wire was displaced towards the tongue, making removal simple.

The operating surgeon and assistants were the same during the whole study. The following parameters were recorded in both the groups:

- Time was recorded in minutes from the beginning of the device fixation till the end of IMF.

- Needle stick injuries, that is, the incidence of perforations in the gloves of surgeon and the first assistant, were identified by the water inflation method of the double gloving system.

- Postoperative occlusion was recorded $24 \mathrm{~h}$ after the surgery, at the first month, and the third month postoperatively as a stable or unstable occlusion, based on Angles classification.

- The Oral Hygiene Index (simplified) given by Greene and Vermillion was used to evaluate the oral hygiene status of the patient after $24 \mathrm{~h}$ of surgery, and recorded as good, fair, or poor.

- Any iatrogenic injury such as tooth avulsion, teeth fracture, interdental papilla striping, and tooth mobility was recorded after completion of the procedure.

- In both groups, gender and age distribution were also recorded.

\section{RESULTS}

In group A, mean age of the patients was 32.04 years in comparison with 29.88 years in group $\mathrm{B}$. The mean age of male patients was 30.27 years, and that of female patients was 41.66 years. The difference between the two groups was statistically significant $(p<0.05)$.
The mean age among all 50 patients was 30.96 years. Of the 50 patients, 47 were male and 3 were female. Group A had 23 males and 2 females in comparison with 24 males and 1 female in group B. The ratio of male to female patients included in this study was 9:1.

The average surgical time taken was 48.08 min for group $A$ and $3.48 \mathrm{~min}$ for group B $(p<0.05)$. Needle stick injuries to operator as well as to the assistants while performing IMF were found for all the 25 patients of group $A$ and for six patients in group $B$. More than four needle stick injuries $(>4)$ while performing IMF were seen in 20 patients in group $A(80 \%)$ and no patient in group B (0\%), and less than four needle stick injuries $(<4)$ while performing IMF were seen in five patients in group A (20\%) and for all 25 patients in group B (100\%). This difference between the two groups was statistically significant $(p<0.01)$.

Occlusion assessment was checked $24 \mathrm{~h}$ after surgery, first month, and at third month, postoperatively.

Postoperatively $24 \mathrm{~h}$ after surgery, all 25 patients had stable occlusions in group A, while 18 patients $(72 \%)$ in group B had stable occlusion and seven (28\%) had unstable occlusions. This difference between the two groups was statistically significant $(p<0.05)$.

Postoperatively one month after surgery, all 25 patients in group A, and 19 patients (76\%) in group B had stable occlusions while six patients ( $24 \%$ had unstable occlusions $(p<0.05)$. Postoperatively three months after surgery, all 25 patients had stable occlusions in group $A$, and 19 patients $(76 \%)$ in group B were stable $(p<0.05)$.

Oral hygiene status according to $\mathrm{OHI}-\mathrm{S}$ in all patients in group $A$ was also significantly better than patients in Group B $(p<0.05)$. latrogenic injuries were seen in eight patients in group A (Five: interdental papilla striping, and three: grade one mobility) and two patients in group B (crown fracture, and avulsion of teeth) $(p<0.05)$.

\section{CONCLUSIONS}

The researchers concluded that the embrasure wire technique for intraoperative IMF provided an effective alternative to the traditional arch bar technique for the treatment of uncomplicated mandibular fractures.

Embrasure wires proved to be a quick, easy, and reliable technique when considered for the minimal or moderately displaced fractures of dentate region of the mandible.

\section{Implications for practice:}

Embrasure wires have been shown to reduce operating times, needle prick injuries, periodontal trauma, and to promote oral hygiene when compared with the Erich bar technique in the dentate patient with a mandibular fracture.

\section{Reference}

1. Satpute AS, Mohiuddin SA, Doiphode AM, et al. Comparison of Erich arch bar versus embrasure wires for intraoperative intermaxillary fixation in mandibular fractures; Oral Maxillofac Surg. 2018; 22: 419-28. 


\title{
2. Comparative study of all-ceramic crowns obtained from conventional and digital impressions
}

\author{
S Berrendero, MP Salido, A Ferreiroa, et al. Clin Oral Invest. 2019; 23: 1745-51.
}

Traditional fabrication of crowns uses a multistage process which involves an indirect technique that requires an impression and subsequent laboratory completion of the restoration. CAD/CAM systems allow for the production of indirect restorations in a single visit.

CAD/CAM stands for computer-assisted design/computer -assisted manufacture. These systems use an optical camera to take a virtual impression by creating a three dimensional image which is forwarded to a software program. This impression results in a virtual cast on which the restoration is designed.

The software then controls a milling process that uses prefabricated blocks of restorative material, either ceramic reinforced composite or all-ceramic material, to produce the restoration. The end result is chairside production of the crown which is then cemented onto the tooth.

Berrendero and colleagues (2019) reported on their study that sought to compare clinical aspects like "marginal fit," "occlusal contacts," "interproximal contact points," "primary retention," and "final selection," of 30 all-ceramic crowns fabricated from both conventional and digital impressions based on Ultrafast Optical Sectioning ${ }^{\mathrm{TM}}$ technology.

The tested null hypothesis was that no significant difference would be found in clinical aspects between crowns fabricated from both impression techniques.

\section{MATERIALS AND METHODS}

Patients in need of a single crown in an asymptomatic posterior tooth were considered for inclusion. Of the 44 patients evaluated, 30 patients ( 21 females, 9 males, aged between 19 and 70 years) were included in the present study.

The exclusion criteria were advanced periodontal attachment loss, signs of bruxism, absence of adjacent or antagonist teeth to the abutment tooth, and marginal preparation located deeper than $1 \mathrm{~mm}$ subgingivally. Subgingival preparations were excluded due to the inability of the optical scanners to record them.

One posterior tooth was prepared for a crown restoration, and two all-ceramic crowns were fabricated for each recruited patient. One crown was made from a conventional impression technique (group C) and another from a digital impression with an intraoral scanner based on ultrafast optical sectioning technology (Trios intraoral scanner, 3Shape, Copenhagen, Denmark) (group D).

Fifteen molars and 15 premolars, 19 in the maxilla and 11 in the mandible, were treated. All patients received the same clinical protocol, carried out by the same experienced prosthodontist.
The crown margin was placed at juxta-gingival level, and in any case not exceeding a subgingival depth of $1 \mathrm{~mm}$. After tooth preparation, a provisional PMMA restoration previously prepared in the laboratory from a wax-up was placed after being readapted using a temporary acrylic-based and finally bonded with a non-eugenol temporary cement (Temp-Bond).

Impressions were taken one week after tooth preparation. The sequence of the impression procedure (silicone versus digital) was randomized by an external operator using a smart phone application. Digital impressions were taken using the intraoral scanner TRIOS $₫$, according to the manufacturer's scanning protocol.

The quadrant of the prepared tooth, the antagonist arch, and the buccal occlusal bite in maximum intercuspation were optically scanned. The captured data were checked for artifacts and were sent online by the scanner itself directly to the dental production centre.

For conventional impression, a vinyl polysiloxane (VPS) material was used in a two-step impression technique (Express 2 Penta Putty ${ }^{\mathrm{TM}}$ as tray and Express 2 Light Body $^{\mathrm{TM}}$ as wash material) in a standard metallic tray. The antagonist arch impression was taken with irreversible hydrocolloid impression material.

Once removed, impressions were disinfected and poured within one hour in type IV plaster. The conventional models were mounted in a semi-adjustable, Arcon type articulator. The upper model was positioned by means of the associated facebow, and all the lower models were mounted in maximum intercuspidation.

The restorations in both groups were all designed and fabricated at the same dental laboratory by the same dental technician.

The clinical evaluation of the crowns was performed through a double-blind study design. The two crowns to be evaluated were presented in two identical white boxes marked with the letter $A$ or $B$ according to a random assignation reflected in an Excel sheet. Only the studio controller knew which box corresponded in each case to the digital or conventional crown. Two external operators evaluated the all-ceramic crowns, without any communication between them.

Four items were assessed:

(1) Marginal fit was checked around the entire preparation perimeter, using probes with defined tip diameters of $150 \mu \mathrm{m}$ to clinically examine the gap size before cementation; (2) Occlusal contacts were verified with $8 \mu \mathrm{m}$ articulating paper; (3) Interproximal contact points were verified with waxed dental floss; and (4) Primary retention was verified with tactile sensation, assuming the presence of passive fit. 
The examiners were calibrated for the assessment of the clinical parameters. Operators checked each variable evaluating its behaviour as eligible, not eligible or indistinct for every crown, and filling in a data sheet.

Then, considering all the variables in total, they had to select which of the two crowns digital or conventional had the best clinical conditions (final selection). All information was registered on a data sheet.

\section{RESULTS}

Sixty all-ceramic crowns in 30 patients were evaluated in the present study. The number of times that each operator selected each type of crown for each variable is shown in the Table below

For the items marginal fit and interproximal contact points, moderate agreement between the two operators was described, and significant differences were found between two study groups.

Although significant differences were described for the variables primary retention and occlusal contacts, the agreement between the operators was fair, and these results were not consistent.

The results for the final selection (clinical selection) obtained a substantial agreement between the two operators, and Pearson's chi-square test showed significant differences between two study groups.

\begin{tabular}{|c|c|c|c|c|c|c|}
\hline Item/Situation & Operator & $\begin{array}{l}\text { Group D } \\
\text { [digital] }\end{array}$ & $\begin{array}{l}\text { Group C } \\
\text { [conventional] }\end{array}$ & Indistinct & Kappa test & $p$ value \\
\hline \multirow[t]{2}{*}{ Marginal fit } & 1 & 15 & 12 & 3 & \multirow[t]{2}{*}{0.58} & \multirow[t]{2}{*}{0.000} \\
\hline & 2 & 18 & 10 & 2 & & \\
\hline \multirow[t]{2}{*}{ Occlusal contacts } & 1 & 14 & 10 & 6 & \multirow[t]{2}{*}{0.34} & \multirow[t]{2}{*}{0.001} \\
\hline & 2 & 9 & 3 & 18 & & \\
\hline \multirow{2}{*}{$\begin{array}{l}\text { Interproximal } \\
\text { contact points }\end{array}$} & 1 & 12 & 9 & 9 & \multirow[t]{2}{*}{0.54} & \multirow[t]{2}{*}{0.000} \\
\hline & 2 & 14 & 6 & 10 & & \\
\hline \multirow[t]{2}{*}{ Primary retention } & 1 & 9 & 9 & 11 & \multirow[t]{2}{*}{0.34} & \multirow[t]{2}{*}{0.008} \\
\hline & 2 & 7 & 9 & 14 & & \\
\hline \multirow[t]{2}{*}{ Clinical selection } & 1 & 18 & 12 & - & \multirow[t]{2}{*}{0.63} & \multirow[t]{2}{*}{0.000} \\
\hline & 2 & 18 & 12 & - & & \\
\hline
\end{tabular}

\section{CONCLUSIONS}

The researchers concluded that clinically, the digital crowns were statistically superior at the interproximal contact points and the marginal fit. For the variables occlusal contacts and primary retention, no differences were found between the two groups.

In most of cases and in a significant way, both evaluators concluded that the digital crowns produced better clinical conditions.

\section{Implications for practice} software and equipment now available, together with the cost and time savings, support a growing view that the digital option offers superior performance over conventional methods still in use.

\section{Reference}

1. Berrendero S, Salido MP, Ferreiroa A. et al. Comparative study of all-ceramic crowns obtained from conventional and digital impressions: clinical findings. Clin Oral Invest. 2019; 23: 1745-51.
The use of digital technology and the recently updated 